\title{
Research on the Design and Application of Xiuyan Manchu Shadow Play Art Language
}

\author{
Li Han, Yue Yang \\ Academy of Fine Arts at Northeast Normal University, Jilin Changchun 130117, China
}

\begin{abstract}
Starting from the historical transformation and modeling evolution of Xiuyan Manchu shadow play art, this paper analyzes the transformation and communication path in the application of network expression design. On the basis of systematically combing the development dilemma of Xiuyan Manchu shadow play art from prosperity to endangered, it is considered that the method of integrating Xiuyan Manchu shadow play art language into the application of network expression design is an effective application way for practical development The broadcasting mode calls for people to pay attention to the disappearing Xiuyan shadow play art.
\end{abstract}

Keywords: Xiuyan Manchu shadow play; Art language; Network expression; Design and Application.

\section{Introduction}

Shadow art, also known as shadow play, is a comprehensive folk art integrating performance, music, literature, painting, paper cutting and dance. Shadow play, as an ancient form of donkey shadow play in China, was selected as the world intangible cultural heritage list in 2012. Xiuyan Manchu shadow play has a history of more than 300 years. Its development and continuation are in the place where Manchu people live for generations. It has been influenced by Manchu folk culture for a long time and has distinct national and regional characteristics. But now with the impact of film, television and the Internet, people have gradually lost their enthusiasm for shadow play. With the birth of network expression, Xiuyan Manchu shadow play art has brought unprecedented development opportunities, making the design and application of Xiuyan Manchu shadow play art in network expression have important practical value and innovative significance.

\section{The Historical Change and Modeling Evolution of Xiuyan Manchu Shadow Play Art}

Xiuyan Manchu shadow play art has experienced four periods, which are formation period, maturity period, prosperity period and atrophy period. In the early stage, the performance structure was called "unique shadow". During the Tongzhi period of the Qing Dynasty, "sliding shadow" gradually formed, and gradually evolved into "flip shadow" in the late period of the Republic of China. Now Xiuyan Manchu shadow still retains part of the original ecological characteristics of "Liukou shadow". From the perspective of historical development, its early form of performance is called "solo" by local folk shadow puppet artists. As the name implies, it is a "solo play" performed by one person. At the end of Qing Dynasty and the beginning of the Republic of China, Xiuyan Manchu shadow play entered a mature period. Folk artists Lianxin Zhao, Dehuai Zhao and Yandong Fang were the representatives. They were called "three famous masters" of Xiuyan Manchu shadow play. They were leading three pillars for a long-term. Therefore, the unique shadow of Xiuyan Manchu shadow play was replaced by the shadow class. In the early days of new China, Xiuyan Manchu shadow play developed rapidly. There were more than 80 artists and 30 boxes of shadow play in the county. The local people would invite shadow play troupes to sing shadow in their spare time. Shadow play was very popular. After the mid-1960s, Xiuyan Manchu shadow play entered a shrinking period under the influence of a special historical period. Many handed down shadow boxes and precious shadow rolls were destroyed. After 1978, Xiuyan cultural workers and shadow play artists began to reform and explore the traditional shadow play.

Manchu's religious has a great influence on the development of Xiuyan Manchu shadow play. Shamanism is widely spread in the north of China,its most basic feature is "all things have spirit", and it thinks that all things in the world are dominated by gods. This kind of deep belief foundation 
makes the "wish making" in Manchu area very popular.After making a vow, they must redeem the vow. In Xiuyan area, we often choose to sing shadow to show a strong sense of ceremony. This is the spiritual element that Xiuyan Manchu shadow play art can always been performed.Artists are deeply influenced by Manchu costumes when they are modeling the movie people. Most of the movie people are dressed in cheongsam inside, with arrow sleeve of mandarin jacket on the outside and a flag bun with four seasons flowers on their heads. This is the dress of Manchu noblewomen. The young girls wore braids, while the male figures wore the official clothes of Manchu in Qing Dynasty, with flower feathers on the top and a braid on the back.Some of them wore melon hats and shoes. These clothes all had strong Manchu, which also formed a unique artistic language of Xiuyan Manchu shadow art.

According to the facts, I visited an inheritor who named ShuilongYu in Tangheling village of Xiuyan Manchu Autonomous County. He is a shadow play artist and the apprentice ofZhengyeLv, the inheritor of Xiuyan Manchu. ShuilongYu not only makes shadow play work, but also likes collecting them. Many shadow play works have a history of more than 100 years, while the walls of his home are full of new shadow play works made by this year.According toShuilongYu's introduction, the materials needed for making shadow play works are all local donkey skins, which can only be processed and made after more than ten processes such as soaking and drying.Put the processed donkey skin on the created pattern. If the skin is thin, put two at the same time. In this way, you can carve two at a time for shadow play making. The most difficult part of making shadow play works is the control of hands and the mastering of carving strength. According to the artist ZhengyeLv, there were three kinds of knife techniques in the making of Xiuyan Manchu shadow puppets earlier, i.e. Throwing Knife, falling knife and force knife. The skill of making force knife has been lost.

\section{Transformation and Propagation Path of Network Expression Design}

With the rapid development of Internet chat tools, wechat, microblog and other instant messaging platforms have gradually become a necessary way of communication, so the network expression came into being, and gradually become the auxiliary communication and expression in chat. In 1982, professor Scott Farman of Carnegie Mellon University in the United States wrote a message on an electronic bulletin: "I suggest that joke tellers can mark '-)' with the following character sequence and look over their heads." So the first emoticon on the Internet is made up of a colon, a hyphen and a bracket, and it has become popular.

In the early stage, network expression was only composed of simple words or punctuation. With the development of technology, it gradually transformed into colorful languages and styles, forming static or dynamic icons, and some screenshots, psgraphs and other design forms processed by software. On the whole, network expression is mainly composed of numbers, punctuation marks, figures and lines. The expression form is simple and clear, and it can vividly and show the rich expressions and actions of the subject, such as the different changes of facial expressions, the movement track of body movements, etc., so as to simplify the process of language understanding and interpretation in communication and interaction.

Due to the popularity of new media such as the Internet and mobile phones, nowadays not only teenagers, including many adults, are using Internet expressions to assist the text, which is effective in expressing meaning in chat. Internet expression has become the most direct reflection of people's emotions and spirits. As a new media communication path, its influence and value can not be ignored. In the instant messaging platform, network expression usually carries complex information and connotation with simple and interesting pictures, which makes it easier for the audience to identify and understand. It adapts to the communication needs of people in modern society and realizes the conversion of emotion and the wide spread of audience. Therefore, how to integrate the modeling features of Xiuyan Manchu shadow play art language with the network expression organically, and how to implement the dynamic expression will be conducive to the broader art dissemination and more efficient network promotion of the traditional folk art, which not only enables people to have a new concept cognition of the traditional art, but also can insert the "wings" of contemporary art into 
the traditional art form Arm "is convenient for artists to further explore the unique style and inner potential of Xiuyan Manchu shadow play art.

\section{Integration and Presentation of Xiuyan Manchu Shadow Play and Network Expression Design}

Extracting the main modeling style of Xiuyan Manchu shadow play, combined with the form of network expression design organically, the traditional modeling elements are retained, and the dynamic details are optimized, including the following two design principles:

First, in the design of decorative patterns, the complex and tedious Manchu costumes are refined artistically to make them conform to the fashion and simple design concepts at home and abroad. The simple and bright design style is not only the fashion trend of today's society, but also the aesthetic appeal of people to the network media. "Minimalism" is not only widely used in the world, but also considered to be the most suitable communication concept for network expression.

Second, in terms of color design, the color system will be optimized for more matching. Although the shadow play itself is bright in color, with large contrast between colors and obvious color difference, in order to conform to the simple design style, the color is optimized as a form of gradient color, and the color is summarized as a more concise two-color matching, which makes the main body more prominent and reduces the color at the same time The contrast and saturation between them can achieve a certain soft color relationship.

In order to better present the Xiuyan Manchu shadow in the network expression, it is necessary to meet the needs of different mobile phones and PC terminals. In terms of the size requirements, it is mainly 750dpi pixels. The outline of the shadow is drawn with the hand written board, and every frame of the shadow's dynamic effect, such as bending, turning and other actions, is drawn, colored and adjusted with the drawing software. Finally, the visual presentation depends on the color matching of the screen. First of all, the use of adjacent colors or homochromatic colors can enhance the unity of network expression language, reflect a sense of stability in the quiet, and form a concise, concise and harmonious visual image. Secondly, complementary color and contrast color can highlight the changes of the picture, and the colors set off each other, clear and eye-catching, which can retain the traditional color matching relationship to the greatest extent. The visual presentation of the dynamic effect is based on the characteristics of the joint movement during the traditional display of Xiuyan Manchu shadow play.

Xiuyan Manchu shadow play itself is the combination of light and shadow, with strong characteristics of two-dimensional space. This study will continue to use the design of twodimensional space, and the current popular flat design style makes the traditional form more prominent. Under the background of the development of new media, the development space and speed of network expression are obvious to all, especially for the spread and development of Xiuyan Manchu shadow play, which has a lot of beneficial effects and promotes the two-way flow with traditional culture. In the current cultural communication environment, network expression breaks the pattern of time and space, and provides a new form of expression for its art communication.

\section{Conclusion}

To sum up, the fundamental research on the design and application of Xiuyan Manchu shadow play art language in network expression is the dissemination of folk traditional culture. Xiuyan Manchu shadow art, born between white mountains and black waters, is not only the representative of Manchu art, but also the excellent model of Chinese traditional culture. In the background of the development of the Internet, the emergence of network expression broadens the communication channels of Xiuyan Manchu shadow play art, breaks the time and space limitations of the traditional folk art, and makes the cultural communication channels more extensive. At the same time, the organic combination of network expression and Xiuyan Manchu shadow play art language, and under the complementary advantages, it has opened up a new innovative development direction, realized 
the deep integration from traditional to contemporary, from art to science and technology, and has a far-reaching impact on the transformation and development of traditional folk art and application research.

\section{References}

[1]. Housheng Luo, et al. Xiuyan shadow play[M]. Liaoning Art Publishing.2014.

[2]. Xiaolin Liu, et al. Investigation and Research on shadow play in South of Liaoning[D]. Shanxi Normal University.2012.

[3]. Wenfang Tan, et al. An analysis of the influence of network emoticons[J].Quest.2011.10. 